\title{
Searching for Double Beta Decay with the Enriched Xenon Observatory
}

\author{
Carter Hall, for the EXO collaboration ${ }^{1}$ \\ SLAC MS 96, 2575 Sand Hill Rd., Menlo Park, CA 94025
}

\begin{abstract}
.
The Enriched Xenon Observatory (EXO) Collaboration is building a series of experiments to search for the neutrinoless double beta decay of ${ }^{136} \mathrm{Xe}$. The first experiment, known as EXO-200, will utilize $200 \mathrm{~kg}$ of xenon enriched to $80 \%$ in the isotope of interest, making it the largest double beta decay experiment to date by one order of magnitude. This experiment is rapidly being constructed, and will begin data taking in 2007. The EXO collaboration is also developing a technique to identify on an event-by-event basis the daughter barium ion of the double beta decay. If successful, this method would eliminate all conventional radioactive backgrounds to the decay, resulting in an ideal experiment. We summarize here the current status of EXO-200 construction and the barium tag $R \& D$ program.
\end{abstract}

Keywords: Double beta decay, Neutrino mass, EXO

PACS: $14.60 . \mathrm{Pq}, 23.40 . \mathrm{Bw}, 23.40 .-\mathrm{s}$

\section{NEUTRINOLESS DOUBLE BETA DECAY}

The search for neutrinoless double beta decay ( $\beta \beta 0 v)$ has recently become a top priority of the global neutrino physics program. As a process which violates total lepton number conservation by two units, $\beta \beta 0 \mathrm{v}$ is our best hope for understanding the Majorana or Dirac nature of the neutrino. This question is directly related to the mechanism which generates neutrino mass in beyond-the-standard-model theories. In addition, the $\beta \beta 0 v$ decay rate can be used to constrain the overall mass scale of the neutrino mass spectrum, providing information complementary to beta decay endpoint measurements, cosmological constraints, and neutrino oscillation studies.

\section{EXO-200}

The Enriched Xenon Observatory (EXO) collaboration [1] is pursuing the development of large scale searches for $\beta \beta 0 v$ based on ${ }^{136} \mathrm{Xe}$. The first experiment in this series, known as EXO-200, is under construction at Stanford University. EXO-200 will contain $200 \mathrm{~kg}$ of xenon enriched to $80 \%$ in ${ }^{136} \mathrm{Xe}$ (already obtained), and will be the largest double beta decay experiment ever attempted.

The EXO-200 detector itself is a liquid xenon TPC, with a central cathode and a detection plane at each end of a cylindrical copper vessel. Charge deposits in the liquid

1 http://www-project.slac.stanford.edu/exo/

Contributed to CIPANP 2006: 9th Conference on the Intersections of Particle and Nuclear Physics, 05/30/2006--6/3/2006, Westin Rio Mar Beach, Puerto Rico 
xenon drift towards the endplates, where the $x$ and $y$ coordinates are measured by a set of crossed wire planes, and where the charge is collected to measure the energy. An array of 259 large area avalanche photodiodes ${ }^{2}$ located behind each pair of wire grids collects the $175 \mathrm{~nm}$ scintillation light, giving a second energy measurement, and also providing a $t_{0}$ measurement for the event. The $t_{0}$ measurement allows us to measure the drift time of the charge deposits, which is converted to the $z$ coordinate by multiplying by the drift velocity. In this manner we achieve full three dimensional reconstruction of the event location, with a position resolution of about $9 \mathrm{~mm}$ in $x$ and $y$ and $6 \mathrm{~mm}$ in $z$. Since multiple site events have interactions which are typically separated by several centimeters, we obtain good rejection of Compton scattered gamma events. Alpha decays are also easy to discriminate against, because the densely ionizing alphas result in a large amount of charge recombination, which can be observed by comparing the charge measurement to the scintillation measurement.

We have studied the energy resolution of liquid xenon in a small experiment where we collected both charge and scintillation. Using a Bi-207 beta source, we found that by taking advantage of an event-by-event anti-correlation between the scintillation and ionization signals, we can achieve an energy resolution of $\sigma(E) / E=3.0 \%$ at $570 \mathrm{keV}$ at a drift field of $3 \mathrm{kV} / \mathrm{cm}$ [2]. Scaling this by $1 / \sqrt{E}$ to our Q value of $2458 \mathrm{keV}$, we project an energy resolution of $\sigma(E) / E=1.4 \%$ for $\beta \beta 0 v$. This represents an improvement of a factor of two relative to the most recent xenon double beta decay experiment [3]. In addition, EXO-200 will have an acceptance for scintillation five times larger than this table-top experiment, which may result in further improvements to the energy resolution.

The copper liquid xenon vessel will be maintained at a temperature $169 \mathrm{~K}$ by a double-walled vacuum-insulated copper cryostat. The inner cryostat has three integrated serpentine heat exchangers, each of which are thermally connected to a refrigerator with vacuum insulated freon transfer lines. The inner cryostat is filled with HFE-7000 cryofluid $^{3}$, which acts as a thermal link between the cryostat and liquid xenon vessel, and also shields the detector from outside radioactivity. The cyrostat was manufactured from low background oxygen free high conductivity copper through electron beam welding at SDMS in Grenoble, France. The cryostat has recently been installed in the EXO cleanrooms at Stanford and will soon undergo its first cooldown test. The cryostat is shielded from environmental radioactivity in the laboratory and rock walls with $25 \mathrm{~cm}$ of high purity lead. The lead has been cast into custom bricks by JL Goslar in Hamburg, Germany.

All materials used to construct the experiment undergo a series of qualification tests. First, materials located inside the lead shield are tested for their ${ }^{238} \mathrm{U},{ }^{232} \mathrm{Th}$, and ${ }^{40} \mathrm{~K}$ content, using a combination of direct gamma counting, neutron activation analysis, and mass spectroscopy. Secondly, all materials in contact with the xenon are tested for outgassing of electronegative impurities such as water and oxygen. These impurities, if dissolved in the liquid xenon, would attenuate the ionization charge as it drifts through the detector, causing the energy measurement to become dependent on the $z$ coordinate. Finally, all materials in the xenon handling system must be tested for radon emission.

\footnotetext{
2 from Advanced Photonix Inc., http: / / www . advancedphotonix. com/

3 from 3M, http: //www. 3m. com/
} 
The collaboration has implemented an online database for tracking materials from the time they are purchased until they are installed in the experiment.

EXO-200 is currently being constructed in a set of six modular clean rooms at Stanford University. In November 2006, the experiment will be shipped inside the cleanrooms to the WIPP facility in Carlsbad, NM, where it will be placed underground at a depth of 2150 feet. Physics data taking is expected to begin in 2007. The projected sensitivity of EXO-200 is listed in Table 1.

\section{BARIUM TAG R\&D}

In addition to developing the EXO-200 experiment, the EXO collaboration is pursuing R\&D towards a system to identify the daughter barium ion from the double beta decay

of ${ }^{136} \mathrm{Xe}$ on an event-by-event basis. If successful, this barium tag technology would eliminate all conventional radioactive backgrounds to $\beta \beta 0 \mathrm{v}$, resulting in an ideal experiment. The method is based upon modern atomic spectroscopy techniques, which allow for the identification and counting of single barium ions in a ion trap.

The spectroscopy of the $\mathrm{Ba}^{+}$ion is characterized by three atomic levels: a ground level $s_{1 / 2}$ state, an excited $p_{1 / 2}$ state, and a metastable $d_{3 / 2}$ state. The $s$ and $p$ states are separated by a $493 \mathrm{~nm}$ (blue) transition, while the $p$ and $d$ states are separated by a $650 \mathrm{~nm}$ (red) transition. When a $\mathrm{Ba}^{+}$ion is illuminated with lasers at these two wavelengths, it rapidly cycles between all three states, in the process scattering laser light in all directions. The scattering rate can be as large as $10^{7}$ photons per second for a single ion, which is large enough to be seen by the naked human eye.

The challenge of the barium tag R\&D is to combine this mature atomic physics technique with a particle physics detector. Currently the EXO collaboration is pursuing two different avenues: 1) identification of the barium ion in situ where the $\beta \beta 0 v$ decay occurs, or 2) transport of the ion from the detector to an ion trap. The in situ method, which could in principle be applied to either a liquid or high pressure gaseous xenon experiment, is appealing simple, but suffers from pressure broadening of the atomic transitions within the xenon medium, requiring large laser power and superb rejection of background scattered light. In the ion transport method, the atomic spectroscopy can occur in the low pressure environment of an ion trap, with a favorable geometry for detecting the scattered light and rejecting ambient backgrounds, at the expense of the additional complication of the transport mechanism.

One transport scheme which we are investigating is an electrostatic probe which could be inserted into the xenon medium at the location of a candidate $\beta \beta 0 v$ event. The ion can be collected by applying a voltage to the probe, and then released into an ion trap designed to accept such a probe. We have shown that a simple tungsten rod is capable of collecting radioactive ions out of liquid xenon, using ${ }^{228} \mathrm{Th}^{+}$and ${ }^{224} \mathrm{Ra}^{+}$ions as a substitute for barium. In addition, we have built a linear ion trap whose far end could accept a probe as an ion source. The linear trap is operational, and has demonstrated single barium ion sensitivity (using a barium oven as a source).

The main remaining hurdle to demonstrating a viable barium tag technology in the ion transport scheme is the issue of ion release from the probe. We are pursuing several potential solutions to that problem, including a cold probe which would trap the ion in 
TABLE 1. Neutrino mass sensitivity of several EXO scenarios.

\begin{tabular}{|c|c|c|c|c|c|c|c|c|}
\hline Case & $\begin{array}{c}\text { Mass } \\
\text { (ton) }\end{array}$ & $\begin{array}{c}\text { Eff. } \\
(\%)\end{array}$ & $\begin{array}{c}\text { Run time } \\
\text { (year) }\end{array}$ & $\begin{array}{c}\sigma(E) / E \\
(\%)\end{array}$ & $\begin{array}{c}\text { b.g. } \\
\text { (events) }\end{array}$ & $\begin{array}{c}T_{1 / 2}^{0 v} \\
(\mathrm{yr}, 90 \% \mathrm{CL})\end{array}$ & $\begin{array}{c}v \text { mass (meV) } \\
\text { QRPA[4] }\end{array}$ & NSM[5] \\
\hline EXO-200 & 0.2 & 70 & 2 & 1.6 & 40 & $6.4 \times 10^{25}$ & 270 & 380 \\
\hline full EXO & 1 & 70 & 5 & 1.6 & 1 & $2.0 \times 10^{27}$ & 50 & 68 \\
& 10 & 70 & 10 & 1 & 1 & $4.1 \times 10^{28}$ & 11 & 15 \\
\hline
\end{tabular}

xenon ice, and release it through sublimation; a hot probe which would boil the ion off a metal surface on the probe; and a field-emission probe which would emit the ion in the large electric field of an FEM tip. This work is rapidly progressing and we expect to have results in the near future.

Neutrino mass sensitivity for several ton-scale scenarios with barium tagging are listed in Table 1.

\section{CONCLUSIONS}

The EXO collaboration is composed of a broad range of nuclear, particle, and atomic physicists. Our first experiment, EXO-200, will be operational soon, and will be among the most sensitive neutrinoless double beta decay experiments ever performed. We also expect that EXO-200 will make the first observation of $\beta \beta 2 v$ in ${ }^{136} \mathrm{Xe}$. Furthermore, we are pursuing the development of an ideal ton-scale double beta decay experiment by taking advantage of the unique atomic physics of the daughter barium ion of the double beta decay. We expect that this R\&D program and the EXO-200 results will come together in a proposal for a large experiment in about two years.

\section{ACKNOWLEDGMENTS}

This work was supported by US-DoE grants DE-FG03-90ER40569-A019 and DEFG29-02AL68086, and DoE contract DE-AC03-76SF00515.

\section{REFERENCES}

1. M. Danilov, et al., Phys. Lett. B480, 12-18 (2000).

2. E. Conti, et al., Phys. Rev. B 68, 054201 (2003).

3. R. Luscher, et al., Phys. Lett. B434, 407-414 (1998).

4. V. A. Rodin, A. Faessler, F. Simkovic, and P. Vogel, Phys. Rev. C 68, 044302 (2003).

5. E. Caurier, F. Nowacki, A. Poves, and J. Retamosa, Nucl. Phys. A 654, 973c-976c (1999). 